Revista de Investigación Universitaria, 2016, Vol. 5 (1): 74-81

ISSN: 2312-4253 (Versión impresa) ISSN: 2078-4015 (Versión digital)

\title{
Programa "Leo y comprendo" para la comprensión de lectura en estudiantes del segundo grado del nivel primaria
}

\section{Program "Leo and I understand" for understanding reading, second grade students of primary level}

\author{
Jaulis Segovia, Isabel Casimira \\ EP de Educación, Facultad de Ciencias Humanas y Educación, Universidad Peruana Unión \\ Recibido el 14 de setiembre del 2015 - Aceptado el 6 de diciembre del 2015
}

\begin{abstract}
Resumen
El propósito de la investigación fue determinar la efectividad del programa "Leo y comprendo" para la comprensión de la lectura en estudiantes del segundo grado del nivel primario de la institución educativa adventista Tercer Milenio de Pucallpa. El tipo de investigación es básica, de enfoque cuantitativo, el diseño es experimental. La muestra estuvo constituida por 23 niños, y por ser una muestra menor de 50 se utilizó la prueba de Bondad de Ajuste a la Curva de Normalidad de Shapiro-Wilk. En cambio para el análisis estadístico, se consideraron datos paramétricos en la preprueba y posprueba (resultando nivel literal: preprueba $-4,087$ y posprueba 0,000 siendo menores que 0,05; en el nivel inferencial: preprueba -3,402 y posprueba 0,000 siendo mayores que 0,05 ; mientras que en el nivel crítico: preprueba $-3,402$ y posprueba 0,000 siendo mayores que 0,05$)$. Asimismo, se aplicó el estadístico de prueba T de student. Todo el cálculo estadístico se dio a través del software Statistical Package for the Social Sciences (SPSS), versión 20.0. Los análisis se realizaron con un 95\% de confianza y un $5 \%$ de significancia de error.
\end{abstract}

Palabras clave: Comprensión de lectura, nivel literal, nivel inferencial, nivel crítico.

\begin{abstract}
The purpose of the research was to determine the effectiveness of the program "Leo and I understand" for reading comprehension in students in the second grade of primary school Adventist Third Millennium Pucallpa. The basic research is quantitative approach, the design is experimental. The sample consisted of 23 children. As the smaller sample of 50 Test Goodness of Fit to Normal Curve Shapiro was used - Wilk, and for statistical analysis, being of parametric data in the pre-test and post-test (resulting literal level: pre test - posttest 4,087 and 0,000 less than 0.05 and the inferential level so: pretest-posttest 3,402 and 0,000 are greater than 0.05) at the critical level: pretest-posttest 3,402 and 0,000 are greater than 0,05) the statistic Student t test was applied. All statistical calculations were given through the Statistical Package for the Social Sciences (SPSS) version 20.0 software. Analyses were performed with 95\% confidence and 5\% significance of error.
\end{abstract}

Keywords: Reading comprehension; literal level; inferential level, critical level.

Correspondencia al autor: email: xabelita_pretty@hotmail.com 


\section{Introducción}

La comprensión de lectura constituye una de las vías principales para la asimilación de la experiencia acumulada por la humanidad. Su enseñanza contribuye al desarrollo intelectual y afectivo del estudiante (Naranjo, 2012). A su vez encontramos diversas definiciones de acuerdo al enfoque y orientación que tienen los autores. Según Cuetos (2002), Valles (2006), cuando hablamos de lectura comprensiva vamos más allá del acto de descifrar el código de la letra impresa; comprender implica extraer significado, esto a su vez requiere un proceso de construcción activa por parte del sujeto, el cual debe utilizar sus conocimientos previos para darle un sentido al texto.

Valles (2006) desde su enfoque cognitivo definió a la comprensión lectora a la luz de dos conceptos. El primero se refiere a la comprensión lectora como un producto que resulta de la interacción entre el lector y el texto. Aquí interviene la memoria a largo plazo, debido a que el resultado de esta interacción (el producto) se guarda en ella para recordar la información leída. El segundo concepto define a la comprensión lectora como un proceso. Por su parte Dijt y Walter Kintsch (citados por Cuetos, 2002) definen a la comprensión lectora como la construcción de un modelo mental, imaginando la situación haciendo uso de las experiencias propias.

Sin embargo, uno de los problemas más emergentes de la educación, es tener estudiantes que no comprenden lo que leen. Según los estudios realizados como la prueba PISA, hasta el 2001, en comprensión de lectura el Perú ocupó el último lugar (63); en el año 2009, de 65 países evaluados, ocupó el lugar 62. Y en la última evaluación del 2012 ocupamos el lugar 66, siendo el puntaje alcanzado en comprensión de lectura 384, lo que posicionó al Perú entre los niveles 1a y 2, el nivel más bajo de todos los países que participaron en la evaluación 2012 (MINEDU, 2013).

Del mismo modo, según el informe mundial de evaluación educativa, realizado por la Organización Educativa Científica y Cultural de las Naciones Unidas (UNESCO) en América Latina y el Caribe, el 40\% de los estudiantes son incapaces de comprender frases simples.

Estos problemas tienen diversos factores, entre los cuales diversas investigaciones han demostrado que el éxito o el fracaso de los estudiantes, está ligado a las habilidades que poseen para leer comprensivamente.
Así, para Morlés (1999), los problemas de comprensión lectora "Son consecuencia de la inexistencia del uso de estrategias apropiadas para desarrollar las habilidades". Otros factores que se identificaron son por falta de motivación en las sesiones de aprendizaje, los estudiantes tienen una escasa percepción visual al mismo tiempo que presentan problemas de memoria de largo plazo (Aylwin \& Muñoz, 2005); y, por otro lado, los padres de familia no se involucran en el acompañamiento de sus hijos en el proceso de enseñanza y aprendizaje (Vivanco, 2014). Estos factores como consecuencia originan que los estudiantes no tengan un buen rendimiento, pero sobre todo les impedirá desarrollar las destrezas cognitivas en cuanto al vocabulario y contenidos que pueden emplear para producir mensajes lingüísticos con fluidez, seguridad y de acuerdo al grado que cursan (Defior, 1998, citado por Pérez, 2010). También tendremos niños desmotivados para comprender textos (Samper, 1993).

En respuesta a esta problemática se ha elaborado el programa "Leo y comprendo", con el fin de mejorar y hacer uso de estrategias lectoras. En cuanto a la teoría interactiva y los procesos que intervienen para su decodificación, y el lector, esto lo explica Isabel Solé (2000) de la siguiente manera: Cuando el lector se sitúa ante el texto, los elementos que lo componen generan en él expectativas a distintos niveles (el de las letras, las palabras), de manera que la información que se procesa en cada uno de ellos funciona como input para el nivel siguiente; así, a través de un proceso ascendente, la información se propaga hacia niveles más elevados. Pero simultáneamente, dado que el texto genera también expectativas a nivel semántico, de su significado global, dichas expectativas guían la lectura y buscan su verificación en indicadores de nivel inferior (léxico, sintáctico, grafofónico) a través de un proceso descendente. Así el lector utiliza simultáneamente su conocimiento del mundo y su conocimiento del texto para construir una interpretación acerca de aquél.

Desde el punto de vista de la enseñanza, las propuestas que se basan en esta perspectiva señalan que los alumnos aprenden a procesar el texto y sus distintos elementos, así como las estrategias que harán posible su comprensión. Según esta teoría, una persona, para leer, necesita dominar la decodificación, pero va más allá porque asume que la persona que lee interpreta el texto, no lo repite de forma mecánica. El proceso interactivo es al mismo tiempo ascendente y descendente. Al respecto, Kenneth Goodman (1982) menciona que el proceso de lectura "debe comenzar con 
un texto con alguna forma gráfica; el texto debe ser procesado como lenguaje; y el proceso debe terminar con la construcción del significado. Sin significado no hay lectura, y los lectores no pueden lograr significados sin utilizar el proceso".

En el proceso de interacción entre el lector y el texto, la persona pone en juego una serie de elementos: la información que facilita el texto, la información que facilita el contexto y los conocimientos previos que el lector posee sobre el texto y sobre el mundo (Bofarull: 2001).

Por el conocimiento cultural que posee, es decir, los conocimientos previos, Goodman afirma que toda lectura es interpretación y que esta depende de lo que la persona ya sabe antes de ejercer esta acción.

En este sentido, las personas de una misma cultura construirán un significado similar pero no el mismo, nadie comprenderá un texto de la misma manera, es decir, de la misma forma que otra persona. De hecho, únicamente se pueden realizar interpretaciones sobre la base de lo que ya se conoce.

Para el modelo interactivo, leer es un proceso en el que interactúan el texto y el lector, en el que tienen la misma importancia tanto los procesos lingüísticos como los culturales. Cuando se habla de experiencias previas nos referimos a los conocimientos anteriores de las personas, o sea, las estructuras de conocimiento previas (Smith: 1983).

El lector es un sujeto activo que casi siempre buscará significado, únicamente en casos muy específicos y casi automáticos no lo hará; por ejemplo, al leer un número telefónico o un número de serie (Solé: 2000). En otros casos, la persona buscará comprender el texto. Los individuos buscarán en sus esquemas de conocimiento, realizarán inferencias, predicciones, seleccionarán la información importante (lo cual depende de la estructura del texto) y no solo se centrarán en palabras y oraciones aisladas (Goodman: 1987).

Finalmente, el modelo interactivo sostiene que la comprensión del texto se alcanza a partir de la interrelación entre lo que el lector lee y lo que ya sabe sobre el tema. Interactúan como referentes el contexto, el texto y el lector (Torres: 1997). Por ese motivo no hay duda de que formar a los estudiantes en la comprensión de lectura en los tres niveles: literal, inferencial y crítico es una de las principales finalidades de la educación.

\begin{abstract}
Método
La investigación es de tipo básica y de enfoque cuantitativo, porque a partir de esta investigación se presentó ampliar en el desarrollo de la comprensión lectora información que ya existió, contextualizándola a un lugar y tiempo específico. Con un enfoque cuantitativo porque presentó características de medición de fenómenos, recolectando datos para el análisis estadístico mediante el instrumento de evaluación "guía de observación”, que permitió medir las estrategias que el docente utilizó para el desarrollo de la comprensión lectora, así como las variables en estudio. Asimismo, la investigación busca probar la hipótesis planteada: Efectividad del programa "Leo y comprendo" en el desarrollo de la comprensión lectora en los estudiantes del segundo grado de la escuela adventista Tercer Milenio de Pucallpa, de los cuales el $52 \%$ era de género masculino y el $48 \%$ de género femenino.
\end{abstract}

Para la recolección de información se utilizó dos instrumentos: una guía de observación y una lista de cotejo (que sirvió de apoyo a la guía, conteniendo los mismos ítems de evaluación), validadas por el juicio de 5 expertos y por el coeficiente de V-Aiken. Para medir la variable de comprensión de lectura se aplicó la Guía de observación, la que constó de 3 partes: las instrucciones, datos generales y 3 dimensiones (para la evaluación de los datos del instrumento: Dimensión 1, nivel literal con 9 ítems; dimensión 2, nivel inferencial, con 4 ítems; dimensión 3, nivel crítico, con 4 ítems, de acuerdo con la operacionalización de las variables). Asimismo, se aplicó los niveles de evaluación: bueno, regular, deficiente. El nivel de confiabilidad del instrumento $(0,630)$ se midió con base en la medida de consistencia de Alfa de Cronbach (por contener escalas).

Por ser la muestra menor de 50 se utilizó la prueba de Bondad de Ajuste a la Curva de Normalidad de Shapiro-Wilk. En cambio para el análisis estadístico, se consideraron datos paramétricos en la preprueba y posprueba (resultando nivel literal: preprueba $-4,087$ y posprueba 0,000 siendo menores que 0,05 ; en el nivel inferencial: preprueba $-3,402$ y posprueba 0,000 siendo mayores que 0,05 ; mientras que en el nivel crítico: preprueba $-3,402$ y posprueba 0,000 siendo mayores que 0,05$)$. Asimismo, se aplicó el estadístico de prueba $\mathrm{T}$ de student. Todo el cálculo estadístico se dio a través del software Statistical Package for the Social Sciences (SPSS), versión 20.0. Los análisis se 
realizaron con un $95 \%$ de confianza y un 5\% de significancia de error.

\section{Resultados}

Los resultados de los análisis descriptivos relevantes para las investigaciones mostradas en la tabla 1, demuestran que el $70 \%$ de los estudiantes tuvieron un nivel deficiente en la comprensión de lectura en el nivel literal antes de la aplicación del programa; por otro lado un $20 \%$ de los mismos tuvieron un nivel regular y un $10 \%$ presentó un nivel bueno. Estos resultados declaran que los estudiantes no leían correctamente ni entendían la información implícita de los textos utilizados en el desarrollo, sin embargo, después de la aplicación del programa, la comprensión de lectura en el nivel literal tuvo un incremento significativo en un $90 \%$ y solo un $10 \%$ de los mismos alcanzaron una comprensión adecuada.

Tabla 1

Comparación de análisis del nivel literal en la comprensión de lectura antes y después del programa.

\begin{tabular}{lllll}
\hline & \multicolumn{2}{c}{ Antes } & \multicolumn{2}{c}{ Después } \\
\cline { 2 - 5 } Niveles & Frecuencia & Porcentaje & Frecuencia & Porcentaje \\
\hline Deficiente & 15 & 70,0 & 0 & 0,0 \\
Regular & 5 & 20,0 & 2 & 10,0 \\
Bueno & 3 & 10,0 & 21 & 90,0 \\
Total & $\mathbf{2 3}$ & $\mathbf{1 0 0 , 0}$ & $\mathbf{2 3}$ & $\mathbf{1 0 0 , 0}$ \\
\hline
\end{tabular}

En cuanto a los resultados de cada dimensión, la tabla 2 muestra los resultados de comprensión de lectura a nivel inferencial en los estudiantes intervenidos antes del programa "Leo y comprendo". Del 100\% de los estudiantes, el 78\% presentó un nivel deficiente en la comprensión inferencial, y el 22\% presentó un nivel regular. Esto significa que los niños no comprenden la información explícita de los textos.

Sin embargo, después de la aplicación del programa se obtuvo resultados favorables en la investigación, pues del 100\% de los estudiantes, el 96\% presentó un nivel bueno en la comprensión inferencial y solo el 4\% un nivel regular. Esto nos dice que los niños mejoraron notoriamente en la comprensión inferencial y que comprendieron la información explícita de los textos.

Tabla 2

Comparación de análisis del nivel inferencial en la comprensión de lectura antes y después del programa.

\begin{tabular}{lllll}
\hline \multirow{2}{*}{ Niveles } & Antes & & Después & \\
\cline { 2 - 5 } & Frecuencia & Porcentaje & Frecuencia & Porcentaje \\
\hline Deficiente & 18 & 78,0 & 0 & 0,0 \\
Regular & 5 & 22,0 & 1 & 4,0 \\
Bueno & 0 & 0,0 & 22 & 96,0 \\
\hline Total & $\mathbf{2 3}$ & $\mathbf{1 0 0 , 0}$ & $\mathbf{2 3}$ & $\mathbf{1 0 0 , 0}$ \\
\hline
\end{tabular}

En cuanto a los resultados que muestra la tabla 3 acerca de la comprensión de lectura a nivel crítico en los estudiantes intervenidos antes del programa "Leo y comprendo", se aprecia que del 100\% de los estudiantes, el $56 \%$ presentó un nivel deficiente, el $40 \%$ presentó un nivel regular y solo el $4 \%$ un nivel bueno. Esto significa que los niños no han desarrollado el juicio, la crítica o el dar una opinión de acuerdo al texto. Sin embargo, después de la aplicación del programa se obtuvo resultados favorables en la investigación, ya que del 100\% de los estudiantes, el $96 \%$ presentó un nivel bueno y solo el 4\% un nivel regular. Esto nos dice que los niños mejoraron notoriamente en el nivel inferencial. 
Jaulis, I.

Tabla 3

Comparación de análisis del nivel crítico en la comprensión de lectura antes y después del programa.

\begin{tabular}{lccccc}
\hline & \multicolumn{2}{c}{ Antes } & & \multicolumn{2}{c}{ Después } \\
\cline { 2 - 3 } \cline { 5 - 6 } Niveles & Frecuencia & Porcentaje & & Frecuencia & Porcentaje \\
\hline Deficiente & 13 & 56,0 & & & 4,0 \\
Regular & 9 & 40,0 & & 1 & 96,0 \\
Bueno & 1 & 4,0 & & 22 & $\mathbf{1 0 0 , 0}$ \\
\hline Total & $\mathbf{2 5}$ & $\mathbf{1 0 0 , 0}$ & & $\mathbf{2 5}$ \\
\hline
\end{tabular}

Para realizar la prueba de las hipótesis general y específicas se formularon las hipótesis nula y alterna de la investigación. Para la hipótesis general se propone que Ho: $\mu 1=\mu 2$ donde la aplicación del programa "Leo y comprendo" no tiene efectividad significativa en la comprensión de lectura en estudiantes del segundo grado del nivel primario de la escuela adventista Tercer Milenio de Pucallpa.

De igual manera Ha: $\mu^{1} \neq \mu^{2}$ donde la aplicación del programa "Leo y comprendo" tiene efectividad significativa en la comprensión de lectura en estudiantes del segundo grado del nivel primario de la escuela adventista Tercer Milenio de Pucallpa. Y según la regla de decisión: se acepta Ho si $\mathrm{p}>\alpha /$ o se rechaza Ho si $\mathrm{p}<\alpha$ siendo que $\alpha$ es igual 0,05 . Y como ya se mencionó previamente se utilizó el estadístico de prueba no paramétrico a aplicarse: el test de los rangos signados de Wilcoxon mediante el cálculo estadístico.

Tabla 4

Prueba de muestras relacionadas.

\begin{tabular}{|c|c|c|c|c|c|c|c|c|}
\hline \multicolumn{6}{|c|}{ Diferencias relacionadas } & $\mathrm{T}$ & $\mathrm{gl}$ & Sig. (bilateral) \\
\hline & \multirow[t]{3}{*}{ Media } & \multirow[t]{3}{*}{$\begin{array}{l}\text { Desviación } \\
\text { típica }\end{array}$} & \multirow[t]{3}{*}{$\begin{array}{l}\text { Error típ. de } \\
\text { la media }\end{array}$} & \multirow{2}{*}{\multicolumn{2}{|c|}{$\begin{array}{l}95 \% \text { intervalo de } \\
\text { confianza para la } \\
\text { diferencia } \\
\end{array}$}} & & & \\
\hline & & & & & & & & \\
\hline & & & & Inferior & Superior & & & \\
\hline $\begin{array}{l}\text { TEST_POS } \\
\text { TEST_PRE }\end{array}$ & 31,000 & 3,908 & 815 & 32,690 & 29,310 & 38,042 & 22 &, 000 \\
\hline
\end{tabular}

Además, el valor de prueba $(\mathrm{p}<0,05)$ fue significativo, resultado que presenta que no hay evidencia suficiente para aceptar la hipótesis nula, por ello se acepta la hipótesis alterna: la aplicación del programa "Leo y comprendo" tiene efectividad significativa en la comprensión de lectura en los estudiantes del segundo grado del nivel primario de la escuela adventista Tercer Milenio de Pucallpa. El mismo procedimiento se dio para las hipótesis específicas de cada dimensión de la variable independiente, programa "Leo y comprendo". Es así como la prueba de hipótesis específica de la dimensión 1, nivel literal, formula que $\mathrm{H}_{0}: \mu^{1}=\mu^{2}$ donde se ve que la aplicación del programa "Leo y comprendo" no tiene efectividad significativa en la comprensión de lectura del nivel literal en estudiantes del segundo grado del nivel primario de la escuela adventista Tercer Milenio de Pucallpa.

De igual manera Ha: $\mu^{1} \neq \mu^{2}$ donde se ve que la aplicación del programa "Leo y comprendo" tiene efectividad significativa en la comprensión de lectura del nivel literal en estudiantes del segundo grado del nivel primario de la escuela adventista Tercer Milenio de Pucallpa. Se aplicó el estadístico de prueba T student por tratarse de datos paramétricos como ya se explicó previamente. Se consideró $\mathrm{n}-1$ grados de libertad (gl) y 1 - $\alpha$ de probabilidad. 
Tabla 5

Análisis de comparación de medidas para muestras relacionadas en el nivel literal.

\begin{tabular}{|c|c|c|c|c|c|c|c|c|c|}
\hline \multicolumn{10}{|c|}{ Prueba de muestras relacionadas } \\
\hline \multirow[t]{3}{*}{ Media } & & \multicolumn{5}{|c|}{ Diferencias relacionadas } & \multirow[t]{3}{*}{$\mathrm{T}$} & \multirow[t]{3}{*}{$\mathrm{gl}$} & \multirow[t]{3}{*}{ Sig. (bilateral) } \\
\hline & & \multirow[t]{2}{*}{$\begin{array}{l}\text { Desviación } \\
\text { típica }\end{array}$} & \multirow[t]{2}{*}{$\begin{array}{l}\text { Error } \\
\text { típ. de la } \\
\text { media }\end{array}$} & \multicolumn{3}{|c|}{$\begin{array}{l}95 \% \text { intervalo de } \\
\text { confianza para la } \\
\text { diferencia }\end{array}$} & & & \\
\hline & $\begin{array}{l}\text { LITE } \\
\text { POST }\end{array}$ & & & Inferior & Superio & & & & \\
\hline Par 1 & LITE PRE & 16,478 & 1,951 & 407 & 17,322 & 15,635 & 40,506 & 22 &, 000 \\
\hline
\end{tabular}

Además, el valor de prueba $(\mathrm{p}<0,05)$ fue significativo, resultado que presenta que no hay evidencia suficiente para aceptar la hipótesis nula: la aplicación del programa "Leo y comprendo" tiene efectividad significativa en la comprensión de lectura del nivel literal en los estudiantes del segundo grado del nivel primario de la escuela adventista Tercer Milenio de Pucallpa.

La prueba de hipótesis específica 2 con base en la dimensión, nivel inferencial, formula que Ho: $\mu^{1}=\mu^{2}$ donde se ve que la aplicación del programa "Leo y comprendo" no tiene efectividad significativa en la comprensión de lectura del nivel inferencial en estudiantes del segundo grado del nivel primario de la escuela adventista Tercer Milenio de Pucallpa. De igual manera se tiene que Ha: $\mu^{1} \neq \mu^{2}$ donde la aplicación del programa "Leo y comprendo" tiene efectividad significativa en la comprensión de lectura en el nivel inferencial en estudiantes del segundo grado del nivel primario de la escuela adventista Tercer Milenio de Pucallpa. Se aplicó el estadístico de prueba no paramétrico: test de los rangos signados de Wilcoxon mediante el cálculo estadístico.

Tabla 6

Rangos obtenidos en la Prueba de Wilcoxon para el nivel inferencial. Análisis de comparación de medias para muestras relacionadas a la ejecución de la estrategia.

\begin{tabular}{llllll}
\hline & & \multicolumn{4}{l}{ Estadísticos de muestras relacionadas } \\
\hline \multirow{3}{*}{ P Par } & Media & $\mathrm{N}$ & Desviación típ. & Error típ. de la media \\
1 & PRE_INFE & 7,22 & 23 & 1,204 &, 251 \\
& POST_INFE & 15,26 & 23 & 2,137 &, 446 \\
\hline
\end{tabular}

El análisis de prueba t para muestras relacionadas evidenció una diferencia significativa en los puntajes de la ejecución de la estrategia de 2,4.

En la tabla 7 podemos observar que los 20 pares de datos no tienen rango positivo, encontrándose 0 pares con datos empatados y 20 rangos negativos, y que el rango promedio es 10,50.

Tabla 7

Prueba de muestras relacionadas.

\begin{tabular}{|c|c|c|c|c|c|c|c|c|c|}
\hline \multicolumn{10}{|c|}{ Prueba de muestras relacionadas } \\
\hline & & \multicolumn{5}{|c|}{ Diferencias relacionadas } & \multirow[t]{4}{*}{$\mathrm{T}$} & \multirow[t]{4}{*}{ gl } & \multirow[t]{4}{*}{ Sig. (bilateral) } \\
\hline & & \multirow[t]{3}{*}{$\begin{array}{l}\mathrm{Me} \\
\mathrm{dia}\end{array}$} & \multirow[t]{3}{*}{$\begin{array}{l}\text { Desviación } \\
\text { típ. }\end{array}$} & \multirow[t]{3}{*}{$\begin{array}{l}\text { Error típ. } \\
\text { de la media }\end{array}$} & \multicolumn{2}{|c|}{$\begin{array}{l}95 \% \text { intervalo de } \\
\text { confianza para la }\end{array}$} & & & \\
\hline & & & & & \multicolumn{2}{|c|}{ diferencia } & & & \\
\hline & & & & & Inferior & Superior & & & \\
\hline \multirow[t]{2}{*}{ Par 1} & CRI_POST - & 8,0 & 1,261 & 263 & 8,589 & 7,498 & 30,602 & 22 & 000 \\
\hline & CRI_PRE & 43 & & & & & & & \\
\hline
\end{tabular}


Por los datos obtenidos en la tabla 7, el valor de prueba $(\mathrm{p}<0,05)$ fue significativo, resultado que presenta que no hay evidencia suficiente para aceptar la hipótesis nula: la aplicación del programa "Leo y comprendo" tiene efectividad significativa en la comprensión de lectura del nivel inferencial en los estudiantes del segundo grado del nivel primario de la escuela adventista Tercer Milenio de Pucallpa.

La prueba de hipótesis específica 3 con base en la dimensión, nivel crítico, formula que Ho: $\mu^{1}=\mu^{2}$ donde se ve que la aplicación del programa "Leo y comprendo" no tiene efectividad significativa en la comprensión de lectura de nivel crítico en estudiantes del segundo grado del nivel primario de la escuela adventista Tercer Milenio de Pucallpa. De igual manera se tiene que Ha: $\mu^{1} \neq \mu^{2}$ donde la aplicación del programa "Leo y comprendo" tiene efectividad significativa en la comprensión de lectura de nivel crítico en estudiantes del segundo grado del nivel primario de la escuela adventista Tercer Milenio de Pucallpa. Se aplicó el estadístico de prueba no paramétrico: test de los rangos signados de Wilcoxon mediante el cálculo estadístico.

\section{Conclusiones}

Después del análisis realizado en los resultados y el sustento de la tesis sobre Efectividad del programa "Leo y comprendo" para desarrollar la comprensión lectora en los estudiantes de segundo grado del nivel primario de la escuela adventista Tercer Milenio de Pucallpa, podemos llegar a las siguientes conclusiones: el nivel de logro en la comprensión de lectura de los estudiantes del nivel primario de la escuela Tercer Milenio mejoró efectivamente, puesto que en la preprueba el $40 \%$ de los estudiantes tuvo un nivel deficiente; por otro lado un $30 \%$ tuvo un nivel regular y el $10 \%$ un nivel bueno. Sin embargo, en la posprueba, los estudiantes superaron el nivel de logro esperado alcanzando el $100 \%$.

El nivel de logro en la comprensión de lectura en el nivel literal de los estudiantes del nivel primario de la escuela Tercer Milenio, mejoró efectivamente, puesto que en la preprueba el $70 \%$ de los estudiantes tuvo un nivel deficiente; por otro lado un $20 \%$ tuvo un nivel regular y el $10 \%$ un nivel bueno. Sin embargo, en la posprueba, los estudiantes superaron el nivel de logro esperado alcanzando el 90\%. El nivel de logro en la comprensión de lectura en el nivel inferencial de los estudiantes del nivel primario de la escuela Tercer Milenio, mejoró efectivamente, puesto que en la preprueba el $78 \%$ de los estudiantes tuvo un nivel deficiente; por otro lado, solo un $22 \%$ tuvo un nivel regular. Sin embargo, en la posprueba, los estudiantes superaron el nivel de logro esperado alcanzando el $96 \%$. El nivel de logro en la comprensión de lectura en el nivel crítico de los estudiantes del nivel primario de la escuela Tercer Milenio, mejoró efectivamente, puesto que en la preprueba el $56 \%$ de los estudiantes tuvo un nivel deficiente; por otro lado un $40 \%$ tuvo un nivel regular y el $4 \%$ un nivel bueno. Sin embargo, en la posprueba, los estudiantes superaron el nivel de logro esperado alcanzando el $96 \%$.

Todo lo antes descrito dio por concluido que existe evidencia de la efectividad del programa "Leo y comprendo" para la comprensión de lectura en estudiantes de segundo grado del nivel primario de la institución educativa Tercer Milenio de Pucallpa, después de su aplicación.

\section{Recomendaciones}

Finalizando la siguiente investigación sugiero algunas recomendaciones de acuerdo a los resultados obtenidos y a las conclusiones que se llegaron luego de la aplicación del programa.

Primeramente, capacitar a los docentes sobre el uso correcto de las estrategias lectoras a fin de mejorar el nivel de la comprensión en los estudiantes. Incentivar a los docentes a que utilicen textos narrativos como cuentos y fábulas que llamen la atención de los niños y los motive a mejorar el nivel de comprensión lectora.

Establecer en todas las prácticas ejercicios pedagógicos que enriquezcan el desarrollo de los tres niveles de comprensión lectora para que los niños tengan una mejora. Fomentar talleres de comprensión de lectura para los estudiantes. Trabajar la comprensión de lectura en todas las áreas y fundamentarla en el área de Comunicación, sobre todo en el desarrollo de sus competencias básicas como escuchar, hablar, leer y escribir, para que los estudiantes aprendan a ser más analíticos a la hora de interpretar cualquier información.

Motivar a los docentes para que incentiven a los estudiantes a realizar prácticas constantes que los haga competentes en la comprensión de lectura como herramienta principal del conocimiento, permitiéndoles así desenvolverse en el exigente mundo actual. 


\section{Referencias}

Álvarez, H. (2010). Programa interactivo para el desarrollo de la comprensión de lectura. 1-29.

Aragón Espinoza, L. (2009). La enseñanza de estrategias metacognitivas para el mejoramiento de la comprensión de lectura. Pensamientos psicológicos, 125-138.

Arriaga Méndez, J. (2010). El desarrollo de la comprensión de lectura a través de nuevos entornos de la lectura. Escuelas de Ciencias de la Educación, 1-17.

Caballero Escorcia, E. R. (2008). Comprensión de textos argumentativos. Antioquía.

Calero, A. (2011). Cómo mejorar la comprensión de lectura. España, Wolters Klower.

DCN. (2009). Diseño Curricular Nacional de la Educación Básica Regular. Lima.

Gonzales Ramírez, O. (2004). Actividad lúdica como estrategia didáctica para fomentar la comprensión de lectura. Pedagógica Nacional.

Gutiérrez Braojos, C. \& Salmerón Pérez, H. (2012). Estrategias de la comprensión lectora. 1-56.

Gutiérrez Valencia, A. \& Montes, R. (s.f.). La importancia de la lectura y su problemática. Revista Iberoamericana de educación, 1-13.

Irene,Y. A. (2012). Trabajos de la titulación. Obtenido de http://repositorio.utn.edu.ec/bitstream/123456789/1444/1/05\%20FECYT\%201440. pdf

Joapanta Qisaguano, I. M. (2010). Guía metodológica para potenciar la comprensión lectora. Técnica de tocopaxi. Ecuador.

Laguna, M. \& Uchuya, M. (2012). Desarrollando el hábito lector. World Vision - Revista teórica y didáctica, 1-45.

Loss, S., \& Metref, K. (2007). Jugando se aprende mucho. España - Turín, Italia.

Margarita, R. (2005). Actividades para mejorar la comprensión de lectura. CEAC Educación.

Ministerio de Educación. (2012). Idioma Extranjero Inglés. Educación Básica. Bases Curriculares 2012. Chile.

Ministerio de Educación Nacional. (S/F). Serie. Lineamientos Curriculares. Idiomas extranjeros. Colombia.
Morán, D. (2010). Estrategias didácticas para la comprensión de lectura. República bolivariana.

Motya, I. M. \& Risueño, A. M. (2007). El juego en el aprendizaje. Buenos Aires: Bonum.

Novack, J. \& Gown, B. (2007). Aprendiendo a aprender. Barcelona: Martínez Roca.

Panduro Isano, I. Y. (2012). Uso de estrategias para mejorar la comprensión lectora. Florencia, Caquetá.

Pérez, E. M. (2010). Dificultades de aprendizaje. Dificultades de la comprensión lectora, 3-10.

Pérez, M. (Julio de 2004). Estrategias didácticas para el aprendizaje significativo del idioma inglés en sexto grado de educación básica. Caracas, Venezuela.

Ruiz Rivas, A. (2007). Estrategias de la comprensión lectora. 1-18.

Sánchez, E., Ortega, J., y Rosales, J. (2008). Cómo mejorar la comprensión de textos en el aula. Comunicación, lenguaje y educación, 1-26.

Santiago Domínguez, D. (2012). Estrategias didácticas para mejorar la comprensión lectora. Pedagógica Nacional. México.

Sanz Moreno, A. (2003). Cómo diseñar actividades de comprensión lectora. Barcelona: Gobierno de Navarra.

Sole, I. (1992). Estrategia de lectura. Barcelona: Garo.

Sole, I. (1999). Enseñanza de la comprensión lectora. Barcelona, España: Moraja.

Suárez, I. M. (2012). Trabajo de titulación (TLEP). Obtenido de http://dspace.udla.edu.ec/bitstream/123456789/1426/1/ UDLA-EC-TLEP-2012-07.pdf

Thoumi, S. (2004). Motivación de la inteligencia infantil. Colombia: Gamma SA. Toapanta Freire, A. E. (2009).

UNESCO (1993). Proyecto principal de educación en América Latina y el Caribe. Oficina Regional de Educación, 1-77

Valdés Aguirre, I. Y. (2012). Obtenido de http://www. dspace.uce.edu.ec/bitstream/25000/271/1/TUCE-0010-53.pdf

Vásquez, M. \& Rodríguez, M. (2012). Influencia de la técnica juego de roles, basado en el enfoque comunicativo, en la expresión oral del idioma inglés. Tesis de Maestría, Universidad César Vallejo, Trujillo. 